\title{
A case of acute retinal pigment epithelitis: spectral domain optical coherence tomography time course and physiopathologic hypothesis
}

This article was published in the following Dove Press journal:

Clinical Ophthalmology

15 September 2010

Number of times this article has been viewed

\author{
Nathalie Puche' \\ Olivier Offret ${ }^{\prime}$ \\ Jean-Antoine Bernard' \\ Francine Behar-Cohen ${ }^{1,2}$ \\ 'Department of Ophthalmology, Hotel \\ Dieu de Paris, AP-HP, Université \\ Paris Descartes; ${ }^{2}$ Inserm UMRS872, \\ Physiopathology of Ocular Diseases: \\ Therapeutic Innovations, Centre de \\ Recherche des Cordeliers, Université \\ Paris Descartes, Paris, France
}

Purpose: To report the time course of retinal morphologic changes in a patient with acute retinal pigment epithelitis (ARPE) using spectral domain optical coherence tomography (SD-OCT).

Methods: A 30-year old man was referred for blurred vision of his right eye after five days that appeared suddenly 15 days after recovery from a flu-like syndrome. SD-OCT was performed immediately, followed by fluorescein and infracyanine angiography at eight days and then at three weeks.

Results: At presentation, a bubble of sub-macular deposit was observed on the right macula with central golden micronodules in a honeycomb pattern. SD-OCT showed an "anterior dislocation" of all the retinal layers up to the inner/outer segment (IS/OS) line and irregular deposits at the OS level together with thickening of the retinal pigment epithelial (RPE) layer. As visual acuity increased, eight days later, the OCT showed reduction of the sub-retinal deposits and an abnormal hyperflectivity of the sub-retinal and RPE layers was observed. The patient showed a positive serology for picornavirus.

Discussion: The acute SD-OCT sections of this patient with ARPE were compared with histological sections of a 35 day old Royal College of Surgeons rat. Similar findings could be observed, with preservation of the IS/OS line and accumulation of debris at the OS level, suggesting that ARPE symptoms could result from a transient phagocytic dysfunction of the RPE at the fovea, inducing reversible accumulation of undigested OS. Picornaviruses comprising enterovirus and coxsachievirus described as being associated with acute chorioretinitis. In this case, it was responsible for ARPE.

Conclusion: We hypothesize that ARPE syndrome results from a transient dysfunction of RPE, which can occur as a post viral reaction.

Keywords: acute retinal pigment epithelitis, picornavirus, SD-OCT

\section{Introduction}

Acute retinal pigment epithelitis (ARPE), also known as Krill's disease, has been described by Krill and Deutman in $1972 .{ }^{1}$ It is characterized by an acute and severe loss of vision in young, healthy adults due to alteration of the macular pigment epithelium. ${ }^{2}$ The disease equally affects males and females, can be either unilateral or bilateral, and usually resolves spontaneously without treatment within a few months. ${ }^{3,4}$ Fundus examination shows delicate darkish macular spots surrounded by a lighter halo-like zone. The acute occurrence of the infection and its self-limiting course has suggested that a viral infection could be the origin of the disease..$^{5,6}$

Being a rare condition, few descriptions of ARPE have been published. We report herein the first spectral domain optical coherence tomography (SD-OCT) images of
Correspondence: Francine Behar-Cohen Department of Ophthalmology, I Place du Parvis, Notre Dame, 75006 Paris, France

Email francine.behar-cohen@crc.jussieu.fr 

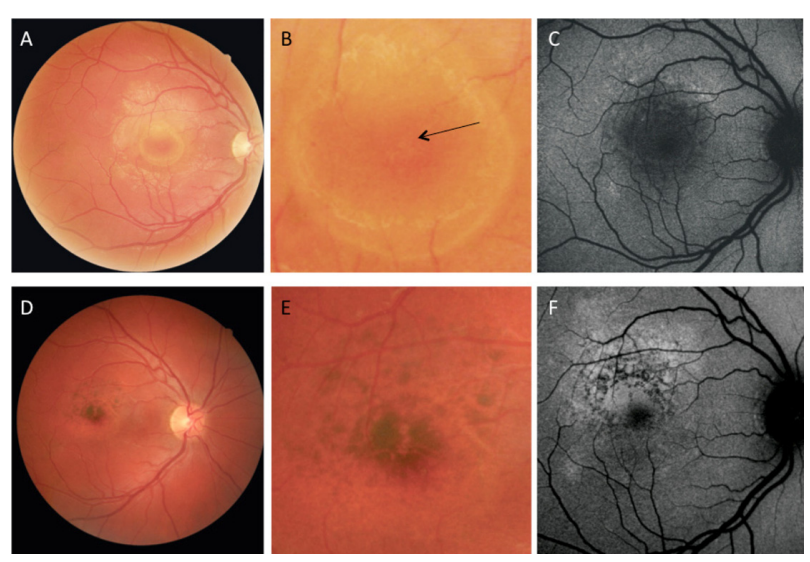

Figure I Color and auto fluorescent fundus photographs. A, B, C: Acute phase of the disease. A) Annular yellowish macular lesions with foveolar pigment irregularity. B) At higher magnification, golden modular lesions in a honeycomb organization (arrow) can be observed in the fovea. C) autofluorescent spots delimitates the macular lesion. D, E, F: Images at three weeks. D) and E) Hyperpigmented deposits are surrounded by hypopigmented halos. F) An autofluorescent shape correlates well with the initial hypopigmented lesion observed on the fundus (A). Pigmented deposits are observed.

an ARPE at the acute and resolving phases. The observation that sub-retinal material accumulates at the level of the outer segment (OS) layer without alteration of the outer limiting membrane (OLM) and inner segment (IS)/OS lines integrity suggests that it may correspond to undigested OS due to transitory dysfunction of the macular retinal pigment epithelium (RPE).

\section{Case report}

A 30-year old man was referred for sudden blurred vision of his right eye from five days prior. Best-corrected visual acuity in his right eye was $2 / 10$ and 10/10 in his left eye. He reported no medical history except recovery from a recent flu-like syndrome fifteen days before. Biomicroscopy of both eyes was unremarkable and intraocular pressure was 11 and $10 \mathrm{mmHg}$ in the right and left eyes, respectively. Ophthalmoscopy showed a circumscribed macular lesion delimited by a circular yellowwhite halo in the right eye (Figure 1A). At higher magnification, golden-colored micronodules in a honeycomb pattern could be observed in the foveal RPE (Figure 1B). On autofluorescent frames, sparse and punctate autofluorescent spots were observed in the upper and temporal border of the annular lesion (Figure 1C). SD-OCT (Spectralis HRA+OCT, Heidelberg Engineering, Heidelberg, Germany) was performed acutely, coupled with angiography at day eight and reproduced three weeks later. At the acute phase, vertical and horizontal sections showed preservation of the foveolar depression and of all retinal layers up to the OLM hyper-reflective line and the IS/OS line (arrow) (Figure 2A). However, a delimitated inhomogeneous thickening of the photoreceptor OS layer was observed under and around the fovea (Figure $2 \mathrm{~A}$ and $2 \mathrm{~B}$, double arrow) resulting in a central macular thickness of $466 \mu \mathrm{m}$ (Figure 3A) together with an irregular rupture of the RPE cells line. This area

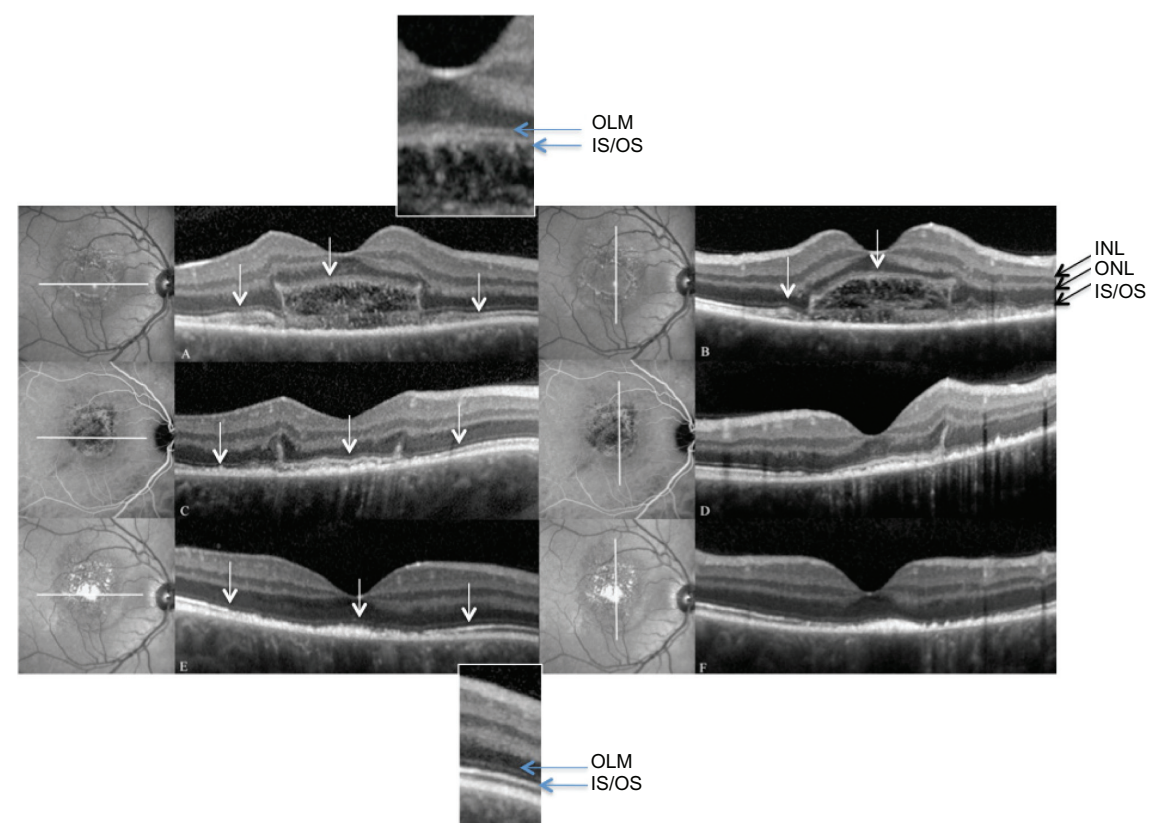

Figure 2 Time course of SD-OCT vertical and horizontal sections. A, B) Acute phase of the disease. All retinal layers above the IS/OS PR line have normal morphology. White arrows represent the IS/OS line. Under the fovea, inhomogenous hyper-reflective fibrillar material form a dome-like deposit. The RPE appears thickened. For better visualization, higher magnification of the fovea is represented showing the ONL and the IS/OS line that are well preserved above elongated and fibrillar OS. C, D) Eight days later, the deposit at the OS/RPE level has almost disappeared except at the border of the lesion. IS/OS line is visible (arrows) and the RPE is irregular and thickened. E, F) At three weeks, the only abnormality is a hyper-reflective dense material at the RPE levels together with a thickening of the RPE line. For better visualization of the ONL and IS/OS line, a higher magnification of the retina is represented.

Abbreviations: INL, inner nuclear layer; ONL, outer nuclear layer; IS, inner segments; OS, outer segments; RPE, retinal pigment epithelial cells. 

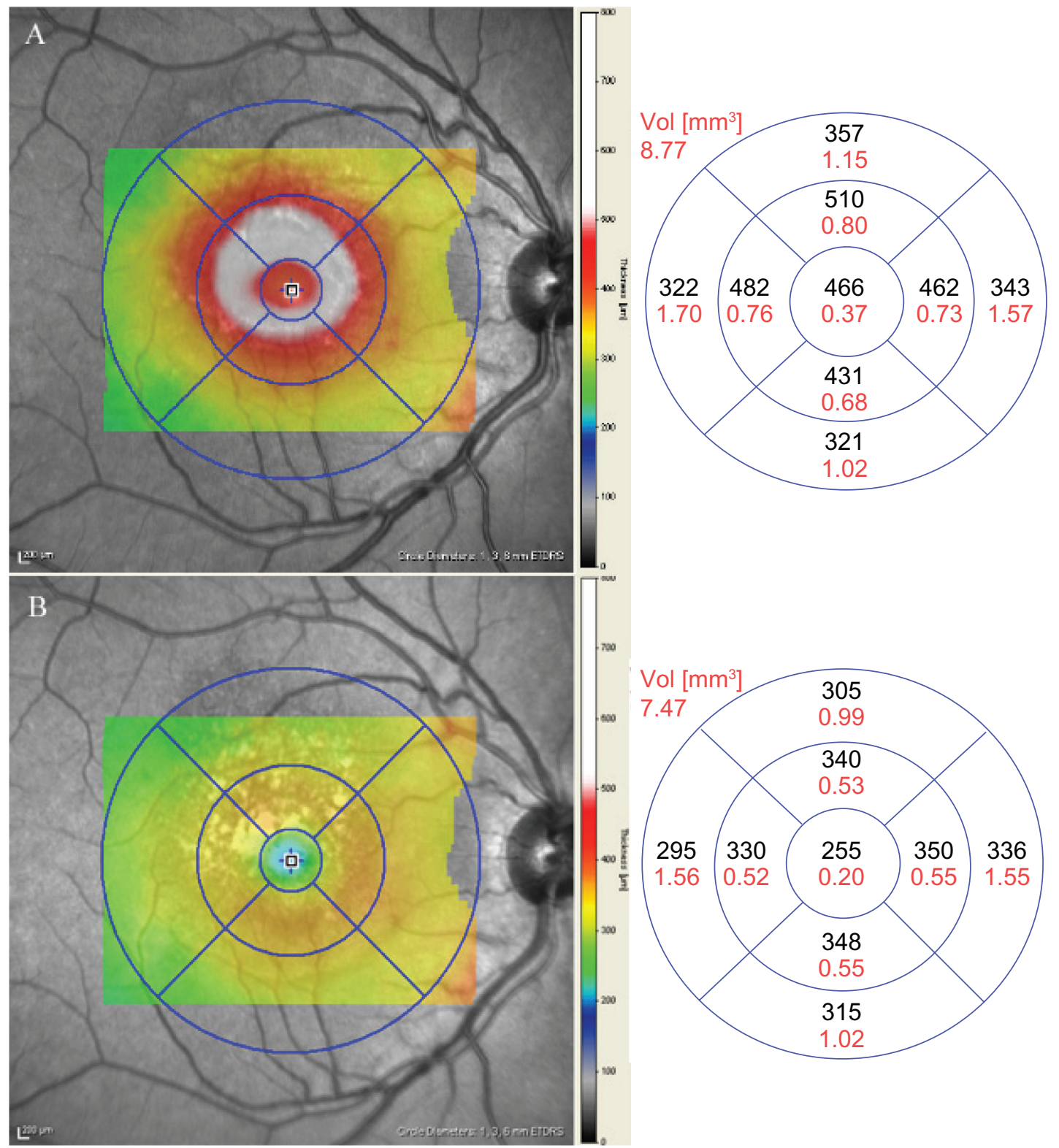

Figure 3 Macular mapping acutely and at three weeks. A) At the acute phase, the dense material under the fovea results in an increased macular thickness. B) At three weeks, the central macular thickness is normal.

seemed delimited by dense vertical lines. No intra-retinal cysts or sub-retinal fluid was observed.

Eight days later, visual acuity progressively recovered up to $7 / 10$ correlating with a striking improvement of retinal morphology on SD-OCT. Indeed, the dense sub-retinal material had resolved leaving only hyper-reflective dense vertical deposits at the border of the lesion inducing focal elevations of the outer nuclear layer (ONL) and the outer plexiform layer (Figure 2C and 2D, arrows). At this stage, the RPE line was reformed even if it appeared irregular and thickened. The IS/ OS line was continuous.

Three weeks later (Figures 2E, 2F, and 3B), central macular thickness was $255 \mu \mathrm{m}$ and retinal sections displayed normal morphology except for a sub-foveolar thickening of RPE and persistence of sub-RPE deposits.

Fluorescein angiography performed at eight days, whilst the sub-retinal deposits were resolving, demonstrated irregular circular transmission hyperfluorescent dots without any leakage at the late phase suggesting alterations of the pigment epithelium (Figure 4A and 4B). The infracyanine angiography on the other hand revealed superposed inhomogeneous deep hypofluorescence (Figure 4C and 4D).

At three weeks, ophthalmoscopy showed hypo and hyperpigmented zones in the macula (Figure 1D and 1E) with well-defined macular pigment stippling with surrounding 


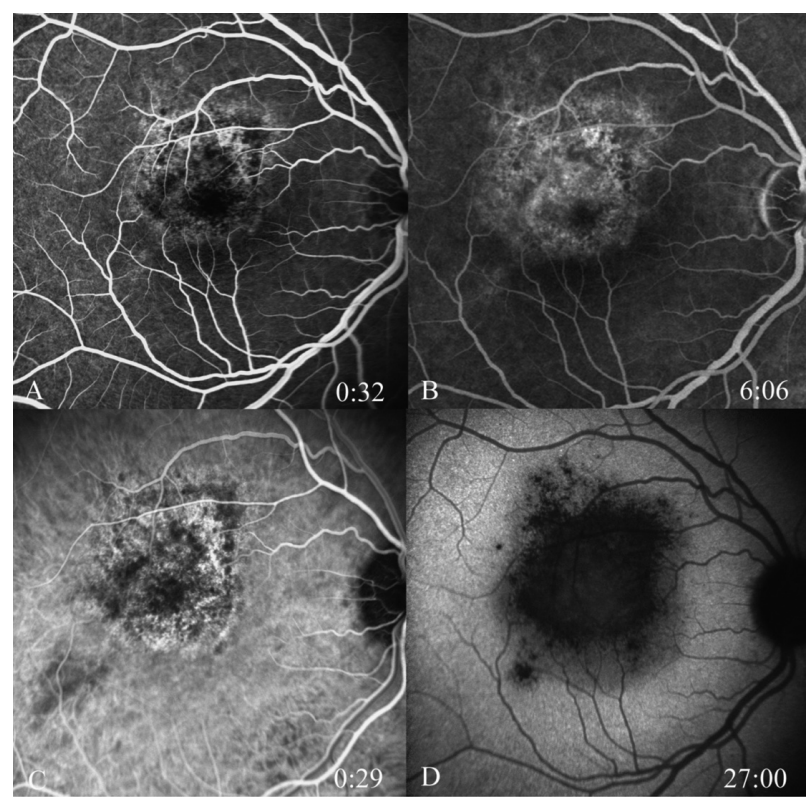

Figure 4 Angiograms at eight days. A, B: Fluorescein early and late phase angiographic images. A) Transmission hyperfluorescence at the border of the macular lesion, B) Impregnation of the RPE. C, D) Infracyanine early and late phase angiographic images. Late hypofluorescence of the entire lesion.

hypopigmentation. Autofluorescent frames showed hyperautofluorescent dots together with pigment masking dots (Figure 1F).

A positive serology for picornavirus was found (4-fold elevation as compared to recovery at three months). The diagnosis of ARPE was retained.

\section{Discussion}

Because ARPE is a rare condition that spontaneously resolves, few cases were reported at the very acute phase of the disease. ${ }^{7-9}$ Hsu described the OCT (OCT3, Carl Zeiss
Meditec Inc, Dublin, CA, USA) findings in three cases of ARPE, performed a few days after the onset of disease. He reported abnormal increased reflectivity involving the ONL and the RPE with absence of intra-retinal, sub-retinal, or sub-RPE fluid suggesting that the initial lesion could involve the foveal photoreceptors. ${ }^{10}$ To our knowledge, this is the first SD-OCT analysis of ARPE at the initial and resolving phases. SD-OCT allows a fine analysis of the outer retinal layers and particularly the IS/OS line, which in this patient remained detectable even at the acute phase when a striking elongation of the OS of the foveolar photoreceptors was observed. The thickening of the OS/RPE layers was strictly localized under the fovea inducing an artifactual "anterior dislocation" of the retinal layers. This localized accumulation of inhomogenous and fibrillar material contrasted with the strict normality of the other parts of the retina. At eight days and three weeks, the OCT images were very similar to those described by Hsu et al in patients with ARPE with hyperreflectivity and densification of the RPE. ${ }^{10}$ The initial macular lesion observed in our patient could correspond either to the very early signs of any ARPE, described as "serous-like macular detachment" by Yoshioka et al. ${ }^{11}$ We have compared the SD-OCT images of this patient to retinal sections of a Royal College of Surgeons (RCS) rat at 35 days of life. Mutation of receptor tyrosine kinase gene, Mertk, in the RCS rat results in defective phagocytosis of photoreceptor OS by the RPE and subsequent retinal degeneration. The disease begins at 20 days of life and at 35 days, and accumulation of undigested OS are observed with preservation of the IS/OS line still (Figure 5A, arrow). At this stage, the undigested OS accumulate in a disorganized manner inducing a thickening of the space between the connecting cilia
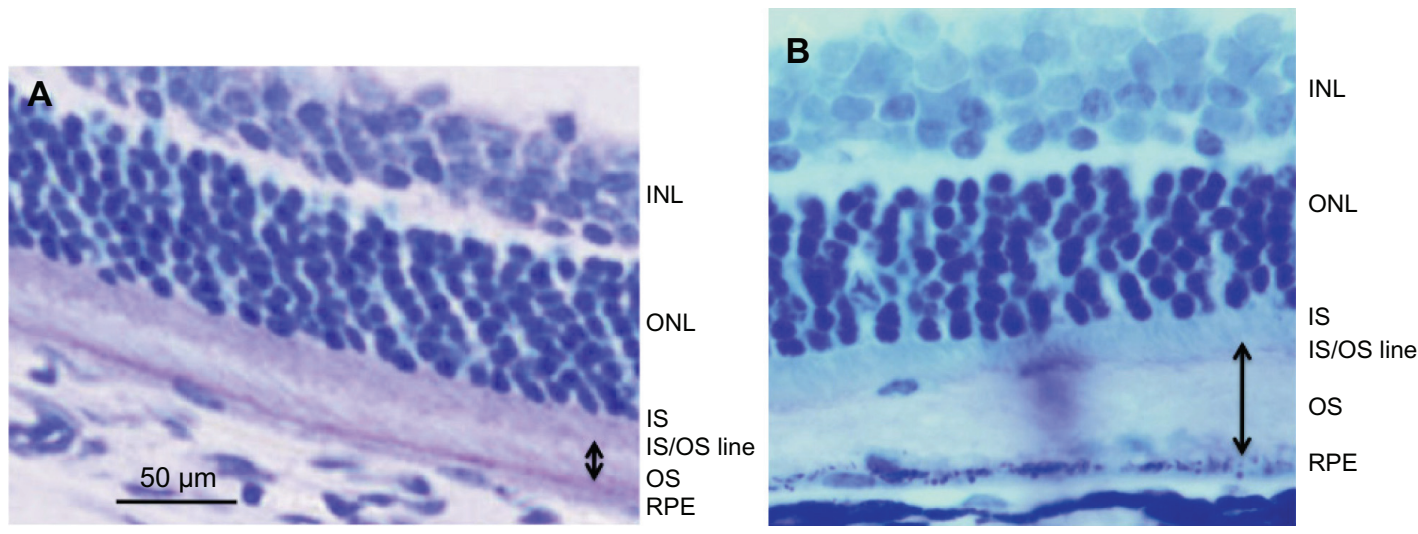

Figure 5 Histology retinal sections of a 35 days old RCS rat. A) Control rat section showing the different retinal layers and the outer segments of photoreceptors (double arrow). B) 35 days old RCS rat section showing preservation of the inner segments and irregular dense undigested outer segments with increased thickness of the OS (double arrow). Dense deposits are also observed above the RPE cells.

Abbreviations: INL, inner nuclear layer; ONL, outer nuclear layer; IS, inner segments; IS/OS line, junction between inner and outer segments; OS, outer segments; RPE, retinal pigment epithelial cells. 
of the photoreceptors and the RPE cells. However, at this time point, when photoreceptors are still alive, the IS and the cilia remain intact explaining the maintenance of the IS/OS line, as observed in our patient. At later stages, as debris accumulates in the RCS rat, and because visual cycle cannot take place, photoreceptors degenerate and the ONL thickness decreases.

Like in the RCS rat, inhomogenous material is observed in our patient at the OS level with preservation of the OLM and IS/OS line on SD-OCT sections. We therefore hypothesize that ARPE symptoms result from a transient dysfunction of RPE cells, with arrest of phagocytosis of the foveal photoreceptors OS.

The reason for this localized accumulation remains unclear. However, the length of the OS and the intense metabolic activity of the cone photoreceptors may partly explain that the RPE dysfunction could be more pronounced at the fovea where RPE cells ingest about 20-30 $\mu \mathrm{m}$ of OS per day.

ARPE has been described as a post viral (C hepatitis virus), ${ }^{5,6}$ but its exact mechanism remains unknown. In our case, the chronology between viral flu-like syndrome and the ocular manifestations, and the positive serology for picornavirus (4-fold increase of antibody rates at the acute phase) suggests that picornavirus may also be associated with ARPE. Picornaviruses comprise enterovirus and coxsachievirus described as being associated with acute chorioretinitis. They may cause respiratory and gastrointestinal symptoms, erythema, meningitis, meningoencephalitis, myocarditis, pericarditis, and myositis. ${ }^{12}$ There have been several reports of coxsachievirus infections associated with chorioretinitis, unilateral acute idiopathic maculopathy, or monofocal outer retinitis and the lesions described bear some resemblance to ARPE. ${ }^{13-16}$ In the present case, the chronology of the ocular symptoms and the systemic viral infection are in favor of a post viral reaction rather than the ocular manifestation of the viral infection itself.

\section{Conclusion}

SD-OCT sections were performed at the acute phase in a patient with ARPE. The morphologic changes suggest that undigested OS may accumulate at the fovea. Similar morphologic changes can be observed in animal models with specific genetic OS phagocytosis deficiency. We therefore hypothesize that an acute and transitory post viral phagocytosis dysfunction of RPE cells may be responsible for ARPE symptoms. The transient character of this disease explains that photoreceptors do not degenerate resulting generally in good visual recovery.

\section{Disclosure}

The authors report no conflicts of interest in this work.

\section{References}

1. Krill AE, AF Deutman. Acute retinal pigment epitheliitis. Am J Ophthalmol. 1972;74:193-205.

2. Eifrig DE, Knobloch WH, Moran JA. Retinal pigment epitheliitis. Ann Ophthalmol. 1977;9(5):639-642.

3. Friedman MW. Bilateral recurrent acute retinal pigment epitheliitis. Am J Ophthalmol. 1975;79(4):567-570.

4. Prost M. Long-term observations of patients with acute retinal pigment epitheliitis. Ophthalmologica. 1989;199(2-3):84-89.

5. Deutman AF. Acute retinal pigment epitheliitis. Am J Ophthalmol. 1974;78(4):571-578.

6. Deutman AF. Acute retinal pigment epitheliitis. Ophthalmologica. 1975;171(4-5):361-364.

7. Jamison RR. Acute retinal pigment epitheliitis with macular edema. Ann Ophthalmol. 1979;11(3):359-361.

8. Luttrull JK, Chittum ME. Acute retinal pigment epitheliitis. Am J Ophthalmol. 1995;120(3):389-391.

9. Luttrull JK. Acute retinal pigment epitheliitis. Am J Ophthalmol. 1997;123(1):127-129.

10. Hsu J, Fineman MS, Kaiser RS. Optical coherence tomography findings in acute retinal pigment epitheliitis. Am J Ophthalmol. 2007;143(1):163-165.

11. Yoshioka H, Sugita T, Okita T, Ryu M. [Serous disciform macular retinal detachment secondary to acute retinal pigment epitheliitis]. Nippon Ganka Gakkai Zasshi. 1979;83(7):818-830. Japanese.

12. Muir P, van Loon AM. Enterovirus infections of the central nervous system. Intervirology. 1997;40(2-3):153-166.

13. Hirakata K, Oshima T, Azuma N. Chorioretinitis induced by coxsackievirus B4 infection. Am J Ophthalmol. 1990;109(2):225-227.

14. Haamann P, Kessel L, Larsen M. Monofocal outer retinitis associated with hand, foot, and mouth disease caused by coxsackievirus. Am J Ophthalmol. 2000;129(4):552-553.

15. Kadrmas EF, Buzney SM. Coxsackievirus B4 as a cause of adult chorioretinitis. Am J Ophthalmol. 1999;127(3):347-349.

16. Beck AP, Jampol LM, Glaser DA, Pollack JS. Is coxsackievirus the cause of unilateral acute idiopathic maculopathy? Arch Ophthalmol. 2004;122(1):121-123.
Clinical Ophthalmology

\section{Publish your work in this journal}

Clinical Ophthalmology is an international, peer-reviewed journal covering all subspecialties within ophthalmology. Key topics include: Optometry; Visual science; Pharmacology and drug therapy in eye diseases; Basic Sciences; Primary and Secondary eye care; Patient Safety and Quality of Care Improvements. This journal is indexed on

\section{Dovepress}

PubMed Central and CAS, and is the official journal of The Society of Clinical Ophthalmology (SCO). The manuscript management system is completely online and includes a very quick and fair peer-review system, which is all easy to use. Visit http://www.dovepress.com/ testimonials.php to read real quotes from published authors. 